\title{
UTILIZAÇÃO DE SIG E ANÁLISE MULTICRITÉRIO PARA SELEÇÃO DE ÁREAS COM POTENCIAL PARA A CONSTRUÇÃO DE UNIVERSIDADES E LOTEAMENTOS UNIVERSITÁRIOS
}

\section{Use of GIS and Analysis Multicriteria for selecting areas with potential for the construction of universities and allotment university}

\author{
Jackeline de Siqueira Castro ${ }^{1}$ \\ Luma Soares Costa ${ }^{1}$ \\ Gustavo Rodrigues Barbosa ${ }^{2}$ \\ Paula Peixoto Assemany ${ }^{1}$ \\ Maria Lúcia Calijuri ${ }^{1}$ \\ Universidade Federal de Viçosa ${ }^{1}$ - UFV.Programa de Pós-Graduação em Engenharia Civil.CEP 36.570- \\ 000 - Viçosa - MG - Brasil (jackeline.castro@ufv.br; luma.costa@ufv.br; paula_assemany@hotmail.com; \\ calijuri@ufv.br) \\ Centro Universitário de Patos de Minas 2- UNIPAM.CEP 38.702-054- Patos de Minas - MG - Brasil \\ gustavorb@unipam.edu.br
}

\section{Resumo:}

A integração de sistemas de informação geográfica com os métodos de decisão multicritério, vem proporcionando inúmeros benefícios para a resolução de problemas de planejamento urbano. Nesse sentido este trabalho apresenta a metodologia utilizada na avaliação e seleção de áreas para a implantação de uma universidade e de um loteamento universitário no município de Patos de Minas - MG. Foram encontrados um local para o loteamento com área de 25,83 ha, e um para a universidade com uma área de 31,47 ha, ambos com risco médio e alta compensação. Os resultados obtidos revelam que o uso da ferramenta SIG associada a análise multicritério são eficientes na escolha de locais para a implantação de empreendimentos que causarão impactos no ambiente a ser instalado.

Palavras-chave: sistema de informação geográfica, análise multicritério, universidade, loteamento universitário.

\section{Abstract:}

The integration of geographic information with the methods of multicriteria decision systems, has provided numerous benefits to solving problems of urban planning. In this sense this work presents the methodology used in the evaluation and selection of areas for the implementation of a 
university and a college allotment in the city of Patos de Minas - MG. A location for the allotment with an area of $25.83 \mathrm{ha}$, and one for the university were found with an area of 31.47 ha, both with medium risk and high reward. The results show that the use of the GIS tool associated with multicriteria analysis are efficient in selecting sites for the implementation of projects that will have impacts on the environment to be installed.

Keywords: geographic information system, multicriteria analysis, university, college allotment.

\section{Introdução}

A escolha do local para a construção de universidades precisa ser melhor explorada do ponto de vista de planejamento urbano, já que causa impactos significativos no ambiente nas quais são instaladas no que diz respeito a modificações no fluxo de veículos, economia local e regional, especulação imobiliária, aumento da população flutuante além da necessidade de produtos e serviços específicos. Além disso, nas proximidades dos locais onde são construídas, pode-se perceber uma expansão urbana expressiva, o que causa também modificações no ambiente e paisagem local.

Apesar do exposto, pouco se sabe sobre estudos realizados pelas instituições públicas e privadas, no que diz respeito à escolha de locais com potencial para a construção de universidades e previsão para a expansão urbana decorrentes da instalação das mesmas.

Segundo Star e Estes (1991), o Sistema de Informação Geográfica (SIG) é considerado uma poderosa ferramenta capaz de integrar o conjunto de rotinas de programação desenvolvidas para representar e manipular grandes quantidades de dados armazenados em bancos de dados, através de coordenadas geográficas, possibilitando assim análises espaciais. Ao se reportar à ferramenta do SIG como tomada de decisão, um dos métodos mais importantes é a análise multicritério. Uma análise de multicritérios é uma ferramenta matemática que permite comparar diferentes cenários, baseada em vários critérios, com o intuito de auxiliar na tomada de decisões, (Roy, 1996 apud Corseuil, 2006), tornando-as mais eficazes.

Segundo Chen e Zhu (2010) neste método, duas técnicas podem ser utilizadas: a análise booleana e a análise de combinação ponderada. $\mathrm{Na}$ combinação ponderada existem as técnicas de Combinação Linear Ponderada (WLC) e a Média Ponderada Ordenada (OWA).

Neste contexto, a utilização da análise multicritério foi realizada, com o objetivo de definir as melhores áreas para construção de centros universitários e concomitantemente locais com potencial para criação de um loteamento universitário.

\section{Materiais e Métodos}

O município de Patos de Minas está localizado no interior do Estado de Minas Gerais, na região do Triângulo Mineiro e Alto Paranaíba, a 417 km da capital do estado, Belo Horizonte. Situado 
nas coordenadas $18^{\circ} 34^{\prime} 44^{\prime}$ ' $\mathrm{S}$ de latitude e $46^{\circ} 31^{\prime} 05^{\prime}$ ' $\mathrm{W}$ de longitude, a uma altitude média de 842 metros de altitude e abrange uma área de $3.189,771 \mathrm{~km}^{2}$.

Para o desenvolvimento da metodologia foram utilizadas as bases topográficas obtidas junto a Prefeitura Municipal deste município no ano de 2012 em escala 1:12.500. As curvas de nível foram extraídas de imagens SRTM projetadas retiradas da EMBRAPA.

No primeiro momento, os dados foram trabalhados no software ArcGIS 10.1 onde todas as informações necessárias para a tomada de decisão, foram reunidas em um único banco de dados, convertidos para a mesma projeção (WGS 1984 - zona 23 S) e só então transferidos para o software Idrisi Taiga, para realização da análise multicritério por meio do método AHP (processo de hierarquia analítica). O AHP é um método de apoio multicritério (MCDM - Multi-Criteria Decision-Making) que procura esclarecer o processo de tomada de decisão, tentando incorporar aos julgamentos, os valores dos agentes, na intenção de acompanhar a maneira como se desenvolvem as preferências (Singh, 2013).

Inicialmente a análise multicritério foi realizada para definir melhores áreas para a instalação de um loteamento universitário, com área mínima de 10 ha. Para este, as restrições foram definidas tendo-se como base a Norma federal sobre parcelamento do solo urbano (Lei 6.766/79), que prevê sua implantação em áreas não passíveis de alagamento, com declividade igual ou inferior a 30\%, que contemplem as exigências ambientais previstas. Além destas, foi estipulado que o loteamento deve estar dentro do perímetro urbano previsto no plano diretor e fora dos bairros, os quais já estão loteados. Já os fatores, foram definidos de acordo com as necessidades do loteamento com vistas a seu bom funcionamento, em termos de acesso, infraestrutura, moradia, como exemplo: quanto mais próximo das vias urbanas melhor e quanto mais plano melhor.

Um cenário WLC foi realizado para o loteamento, com risco médio e compensação 1. A melhor área encontrada para a construção do loteamento foi utilizada como fator para a análise multicritério para definição de áreas para a construção de universidades, que requer uma área mínima de 30 ha.

As restrições utilizadas para a escolha de áreas para universidade foram: a área doada deverá ser contínua, isto é, não poderá ser dividida por hidrografia, deve estar em um raio de até 10 (dez) quilômetros do centro da cidade, adotando-se como ponto de partida (marco zero para a aferição) o Palácio Dona Filomena de Macedo Melo, antiga sede da Prefeitura Municipal; declividade média suave não superior a $6 \%$ e nem inferior a $2 \%$; superfícies com, no máximo 5\%, de área alagadiça em qualquer época do ano e ausência de restrições legais ambientais para a implantação das estruturas físicas necessárias à instalação do campus. Além disso, a universidade deve estar dentro do perímetro urbano previsto no plano diretor.

Já os fatores foram: quanto mais próximos das vias pavimentadas melhor, quanto mais distante das universidades já existentes melhor, quanto menos declivoso melhor, quanto mais próximo do centro da cidade melhor e quanto mais próximo do loteamento encontrado na análise anterior. $\mathrm{Na}$ ferramenta Decision Wizard no software Idrisi Taiga, os mesmos foram avaliados seguindo a lógica fuzzy atribuindo-se aos fatores, pesos variando de 0 a 255 e considerando que, quanto menor o peso, menor adequabilidade. Os valores de importância para os fatores foram obtidos a partir da matriz de comparação par a par pelo método AHP (Processo Hierárquico Analítico), proposto por Saaty (1980). Na construção de uma matriz de comparação par a par, cada um dos fatores é avaliado contra todos os outros. Sendo que quando o fator localizado no eixo vertical for mais importante do que o fator no eixo horizontal, o valor dado a ele deve variar entre 1 e 9 de acordo com o seu grau de importância. Caso contrário, o valor deve variar entre 1/2 e 1/9. Logo, foram calculados um conjunto de pesos e a razão de consistência (CR - consistency ratio). Quando o valor da CR for menor que 0.1 trata-se de uma boa consistência, valores acima de 0.1 indicam que a matriz requer revisão (Chen e Zhu, 2010). 
Para a universidade foram gerados três cenários sendo eles: um WLC (risco médio e compensação alta), um cenário OWA 1 (risco baixo e alta compensação) e um OWA 2 (risco alto e baixa compensação).

\section{Resultados e Discussão}

A área com maior potencial para criação do loteamento universitário possui $258300 \mathrm{~m}^{2}$ e está localizada na porção noroeste do município.

Com relação aos três cenários (WLC, OWA 1 e OWA 2) realizados para a escolha do melhor local para construção de universidades, as áreas encontradas foram as mesmas para os dois cenários OWA, mesmo tendo variado o risco e a compensação de uma para outra. Para o cenário WLC a área encontrada esteve próxima dos cenários anteriores, porém com área menor $\left(314684 \mathrm{~m}^{2}\right)$ compatível com a exigida no planejamento inicial que previa área mínima de $300000 \mathrm{~m}^{2}$.

Por fim as melhores para a construção da universidade e do loteamento universitário foram definidas de acordo com as exigências por áreas e proximidade entre elas como na figura 1, que segue: A distância entre o loteamento e a universidade seria de 651 metros o que torna viável a implantação dos mesmos neste local.

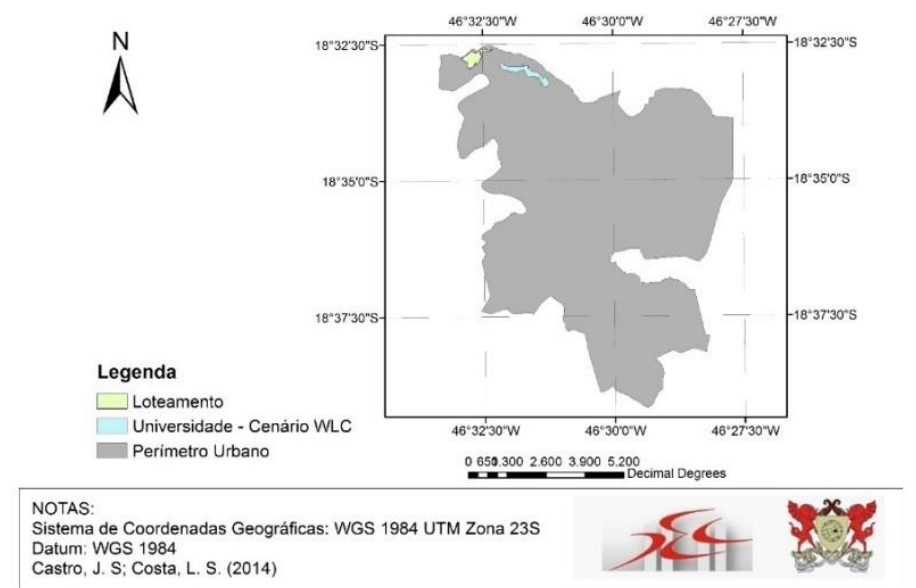

Figura 1: Cenário final com melhor local para a construção da Universidade e do loteamento universitário.

Diversos autores têm investido em análise multicritério para escolha de locais para a construção de diversos cenários. Como exemplo disso, podemos citar Rikalovic et al. (2014), que através de uma combinação de ferramentas, objetivaram a eficiência e qualidade da análise espacial para a escolha de um local para a construção de uma área industrial na região de Vojvodina na Sérvia.

Além destes, podemos citar Sahnoun et al. (2012), que realizaram uma análise multicritério para selecionar potenciais locais para a criação de um complexo agroindustrial na cidade de Sfax (costa sul da Tunísia) onde foram selecionados três locais apropriados.

Em outra situação Hossain et al. (2009), utilizaram a análise multicritério para identificar locais adequados para criação de carpas em corpos d'água urbanos de Chittagong, Bangladesh, com análises voltadas para água, solo e banco de dados de infraestrutura.

Em todos os casos, os autores ressaltam a importância da análise multicritério para a otimização da escolha de locais apropriados para a construção de infraestruturas, utilizando uma análise mais 
ampla, que leva em consideração diversos fatores, contribuindo para o sucesso no funcionamento dos empreendimentos em construção.

\section{Conclusões}

O uso da ferramenta SIG se mostrou eficiente quando da escolha de locais com potencial para construção de loteamentos e universidades. Tais estudos são passíveis de serem realizados para qualquer região, com posse da base de dados adequada. Para este caso, foram encontradas áreas que atendam a todas as exigências estipuladas anteriormente, o que auxilia o analista na tomada de decisão.

Essa análise pode ser refeita utilizando outros softwares, ou mesmo realizando um estudo diferenciado para as restrições e os fatores, podendo gerar novos e talvez mais precisos resultados. Além disso, a utilização de uma base de dados em menor escala pode gerar um resultado mais consistente.

Por fim, pode-se concluir que a análise multicritério pode facilmente ser relacionada a gestão do território e mais ainda com a melhor utilização do recurso público, a fim de que gastos excessivos ou desnecessários sejam realizados.

\section{REFERÊNCIAS BIBLIOGRÁFICAS}

BRASIL. Lei no. 6.766, de 19 de Dezembro de 1979. Dispõe sobre o parcelamento do solo urbano e dá outras providências. Diário Oficial da União, Brasília, 1979.

Chen, Jing and Zhu, Qingjie. "Uncertainty and decision strategy analysis of GIS-based ordered weighted averaging method." International Conference on Information Networking and Automation (ICINA), 2010. Vol. 1. IEEE, 2010.

Corseuil, Cláudia Weber. "Técnicas de geoprocessamento e de análise de multicritérios na adequação de uso das terras". Tese de doutorado. UNIVERSIDADE ESTADUAL PAULISTA “JÚLIO DE MESQUITA FILHO”, 2006.

Hossain, Shahadat M.; Chowdhury, Sayedur Rahman; Das, Nani Gopal; Sharifuzzaman, S. M. and Sultana, Abida. "Integration of GIS and multicriteria decision analysis for urban aquaculture development in Bangladesh". Landscape and Urban Planning 90 (2009): 119-133.

Prefeitura Municipal de Patos de Minas, 2014. Disponível em: http://www.patosdeminas.mg.gov.br. Acessado em 22 de maio de 2014.

Rikalovic, Aleksandar; Cosic, Ilija and Lazarevic, Djordje. "GIS based multi-criteria analysis for industrial site selection." Procedia Engineering 69 (2014): 1054-1063.

Saaty, Thomas L. "The analytic hierarchy process: planning, priority setting, resource allocation". New York: Mcgraw-hill. 1980.

Sahnoun, Houda; Serbaji, Mohamed Moncef; Karray, Boubaker and Medhioub, Khaled. "GIS and multi-criteria analysis to select potential sites of agro-industrial complex". Environmental Earth Science 66 (2014): 2477-2489. 
Singh, Rajesh K. "Prioritizing the factors for coordinated supply chain using analytic hierarchy process (AHP)". Measuring Business Excellence 17 (2013): 80-97.

Star, Jeffrey and Estes, John. "Geographic Information Systems: an introduction”. New Jersey: Englewoods Cliffs, 1991.

Recebido em dezembro de 2014. Aceito em março de 2015. 